\title{
Karyotype and Mechanism of Sex Determination in Ephedra foliata Boiss. - A Dioecious Gymnosperm
}

\author{
P. N. Mehra and Subhash C. Khitha \\ Department of Botany, Panjab University, Chandigarh, India
}

Received June 26, 1979

The genus Ephedra consists of shrubby broom-like plants with extensively developed root system and minute scaly leaves. Photosynthesis, hence, is carried out by the green ribbed stems.

The genus comprises about 35 species distributed almost equally between the Old and New worlds. From the Indian subcontinent 5 species have been recorded, namely, E. foliata Boiss., E. intermedia Shrenk et Mey, E. gerardiana Wall., E. sexatilis Royle and E. nebrodensis Stapf.

Mehra $(1934,1946)$ studied the cytology of 7 species of the genus, two of which, namely, $E$. foliata and $E$. gerardiana, were found to be diploid with $\mathrm{n}=7$ and the rest five tetraploid with $n=14$ chromosomes. He established that the basic karyotype of the genus consists of 5 chromosomes with median-submedian primary constriction and the remaining 2 chromosomes with sub-terminal primary constriction. The two diploid species, E. foliata and E. gerardiana, were reported to be strictly dioecious, while, among the tetraploids, especially in $E$. intermdia, male, female, and hermaphrodite individuals bearing $\hat{\delta}$ and $\phi$ cones were observed.

The first report of chromosome-based sex-determination in plants was made by Allen (1917) on Sphaerocarpos-a liverwort. Subsequently, chromosomes associated with sex have been discovered in a number of dioecious plants, lower as well as higher. In dioecious gymnosperms very little work has been done on the cytology of sex-determination which perhaps may be because the male and female individuals are identical in appearance and vegetative morphology. Eichhorn (1928) was the first to report the existence of satellites in Ginkgo biloba and suggested that these might be related to sex determination. However, Lee (1954) and Pollock (1957) presented evidence of association of satellite-based heteromorphic chromosome pair with sex. Similar observations were made in Cycas pectinata by Abraham and Mathew (1962). An XY type of sex mechanism has been assumed to be present in these plants.

E. foliata Boiss. belongs to the section Pseudobaccate, tribe Scadentes. It is a native of Rajasthan and also occurs in the districts of Haryana State bordering Rajasthan. The plant is strictly dioecious.

In order to elucidate if sex chromosomes are involved in the determination of sex in the species extensive karyotypic analyses were made of the male and female gametophytes, stem apices of male and female plants, root tips of seedlings and meiosis in the pollen mother cells. There is a strong indication that a heteromorphic sex pair is present in this species, and convincing evidence has been furnished to show 
that the male is the heterogametic sex and female is homogametic, and that an XY type of sex mechanism seems to occur in this species also.

\section{Material and methods}

The material for meiotic and mitotic preparations was collected from Botanical Gardens of the Panjab University, Chandigarh, where a large number of plants (male and female) of E. foliata are growing nicely. The male cones, young ovules and stem tips were collected and fixed for cytological studies as per schedule given below:

\begin{tabular}{llll}
\hline & Material & Months of collection & Time of fixation \\
\hline 1. & Male cones & October-November & $12.00-14.00 \mathrm{hrs}$ \\
2. Young ovules & November-December & $11.00-12.00 \mathrm{hrs}$ \\
3. Stem-tips & December & $10.00-11.00 \mathrm{hrs}$ \\
\hline
\end{tabular}

The fixation of material for a study of meiosis was done overnight in Carnoy's fluid (absolute alcohol, chloroform and glacial-acetic acid in the ratio of 9:3:1 respectively) and then transfered to $70 \%$ alcohol. Smears were made in Belling's iron-aceto carmine, and the slides were made permanent by passing them through the following series of grades of glacial acetic acid and absolute alcohol, $1: 1,1: 3$, 1:6, 1:9, absolute alcohol and finally mounted in Euparal.

Young ovules were used for karyotypic studies of the female gametophyte. Sterile scales surrounding the developing ovules were removed before fixing in Farmer's fluid (absolute alcohol and glacial acetic acid in the ratio of 3:1). After fixation overnight they were transfered to $70 \%$ alcohol. The gametophytes were carefully removed from the ovules after $24 \mathrm{hr}$ and transfered to watch glass containing 9 parts $1 \%$ acetic-lacmoid and 1 part $1 \mathrm{~N} \cdot \mathrm{HCl}$. The watch glass was lightly warmed over a spirit lamp and allowed to stand for 5-10 minutes before the gametophytes were squashed in a fresh drop of $1 \%$ acetic-lacmoid. The latter stain was prepared according to the method of LaCour (1942). The slide and the cover-slip were separated in a petri-dish containing normal butyl alcohol ( $\mathrm{N}$-butanol) and mounted in Euparal. Lacmoid stain was found to be ideally suited for a study of mitoses in the female gametophyte.

Fresh viable seeds were germinated on a moist wad of cotton in petri-dishes. Young root-tips were pre-treated in $0.004 \mathrm{M}$ 8-hydroxyquinoline for 3-4 hours, then rinsed quickly in water, and fixed in Farmer's fluid for 24 hours. Root-tips were hydrolysed in $1 \mathrm{~N} \cdot \mathrm{HCl}$ for 10 minutes at $60^{\circ} \mathrm{C}$, then squashed in $1 \%$ Belling's aceto-carmine and finally stained in Feulgen. The same procedure was adopted for a study of stem-apex mitoses of mature male and female plants.

For a study of the karyotype of male gametophyte, mature male cones were fixed in Carnoy's fluid and stained by Warmke's method i.e. the pollen grains were immersed in equal parts of conc. $\mathrm{HCl}$ and $95 \%$ alcohol for 5 minutes without warming. They were then hardened in Carnoy for 5 minutes before staining in Belling's iron aceto-carmine. The slides were made permanent in euparal. 
In determining the morphology of the chromosomes the terminology of Levan, Fredge and Sandberg (1964) is followed i.e. a chromosome with median point centromere giving a value of r-index 1 is designated strictly Metacentric (M); a median region centromere giving r-index value $1-1.7$ is designated as metacentric $(\mathrm{m})$. Similarly the chromosomes bearing sub-median region and sub-terminal region centromere were designated as sub-metacentric (sm) and sub-telocentric (st) respectively.

All the photomicrographs were made at a uniform magnification of $\times 2490$.

\section{Observations and results}

The karyotype of $E$. foliata Boiss. was analysed from four different sources of somatic tissues viz. female gametophyte (endosperm), pollen grains, root-tips, and stem-tips of male and female plants. Male meiosis was studied to gather further evidence for the occurrence of a possible heteromorphic sex pair in the male individuals.

\section{Karyotype from female gametophyte (endosperm)}

The female gametyphyte is an ideal material for karyotypic studies because of the fact that in gymnosperms this tissue has haploid number of chromosomes, unlike the angiosperms where the similar tissue is triploid in origin.

The somatic number as studied from squashes of female gametophyte is $n=7$ chromosomes (Figs. 1 and 2). They are classified on the basis of r-index (see Table 1). The karyotype is of the asymmetrical type with the largest chromosome $12.6 \mu$ and the smallest $8.4 \mu$ in length. The total chromatin length in the haploid set at metaphase is $77.46 \mu$.

Three of the 7 chromosomes i.e. B, D and E are with median region primary constriction (m), one chromosome i.e. $\mathrm{C}$ with median point primary constriction $(\mathrm{M})$, one chromosome i.e. A with submedian region primary constriction (sm) and two chromosomes i.e. $F$ and $G$ are with subterminal (st) primary constriction. Of the seven chromosomes two possess satellites. The chromosome $\mathrm{C}$ which is strictly metacentric (M) has two satellites one on each arm, whereas the other i.e. D which is metacentric (m) has only one satellite located on its short arm. The karyogram of the haploid set is shown in Fig. 8. Chromosome A possesses one secondary constriction in its long arm.

\section{Karyotype from male gametophyte (Pollen-grain)}

The haploid chromosome number as revealed from pollen mitoses is also $\mathrm{n}=7$ (Figs. 3 and 4). In the figures the body nucleus is at the mitotic metaphase. The karyotype is of the asymmetrical type with the largest chromosome $10.5 \mu$ and the smallest $4.8 \mu$ in length at metaphase. The total chromatin length of the haploid set at metaphase is $50.7 \mu$. The basic karyotype is the same as described above with the chromosomes $\mathrm{B}, \mathrm{D}$ and $\mathrm{E}$ having median region primary constriction $(\mathrm{m})$, chromosome $\mathrm{C}$ with median point $(\mathrm{M})$ primary constriction, chromosome $\mathrm{A}$ with submedian region (sm) primary constriction and $F$ and $G$ chromosomes with subterminal (st) region primary constriction. Chromosome A possesses a secondary 
constriction in its long arm.

But, a critical study of the numerous dividing body nuclei revealed a point of difference in the number of satellites on the Metacentric chromosome C. In nearly half the grains this chromosome possessed two satellites one on each arm (Fig. 3), whereas in the others a satellite was observed only on one of the two equal arms (Fig. 4). However, the chromosome $D$ which is metacentric (m) possessed one satellite on its shorter arms in all the pollen preparations.

Table 1. Measurements of somatic chromosomes of Ephedra foliata Boiss.

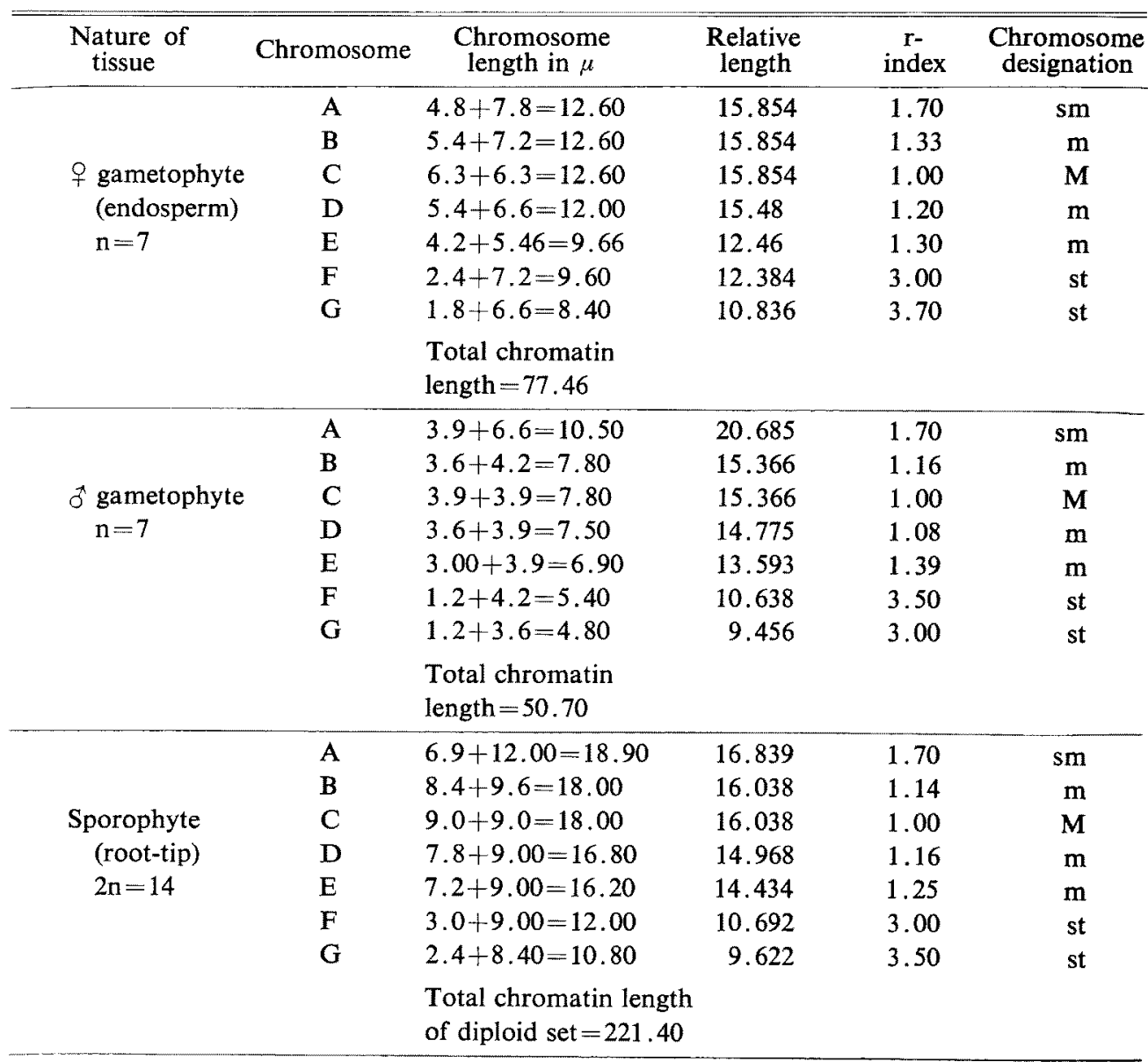

Symbols: M-strictly metacentric; m-metacentric; sm-submetacentric; st-subtelocentric.

Karyotype from root-tips

The somatic number as studied from squashes of root-tips is $2 n=14$ chromosomes. These 14 chromosomes have been classified on the basis of r-index (cf. Table 1). The karyotype consists of five pairs of long and two pairs of short chromosomes. The largest chromosome pair in the complement is of $18.90 \mu$ and the smallest pair $10.80 \mu$ in length. The total chromatin length of the diploid somatic set at metaphase is $221.40 \mu$.

Of the seven pairs of chromosomes, three pairs i.e. BB, DD, and EE possess 
median region $(\mathrm{m})$ primary constriction, one pair i.e. $\mathrm{CC}$ is with Median point $(\mathrm{M})$ primary contriction, two pairs i.e. FF and GG are with subterminal (st) region primary constriction. Of the two satellited pairs one pair i.e. pair CC is strictly Metacentric (M) and is homozygous for satellites in some root-tips, while in others this pair was found to be heterozygous for the satellites. In the heterozygous condition one homologues bears one satellite on each of its arms, while the other one bears a satellite on one arm only. Chromosomes of the DD pair were seen to possess a single satellite on the shorter arms in all the preparations.

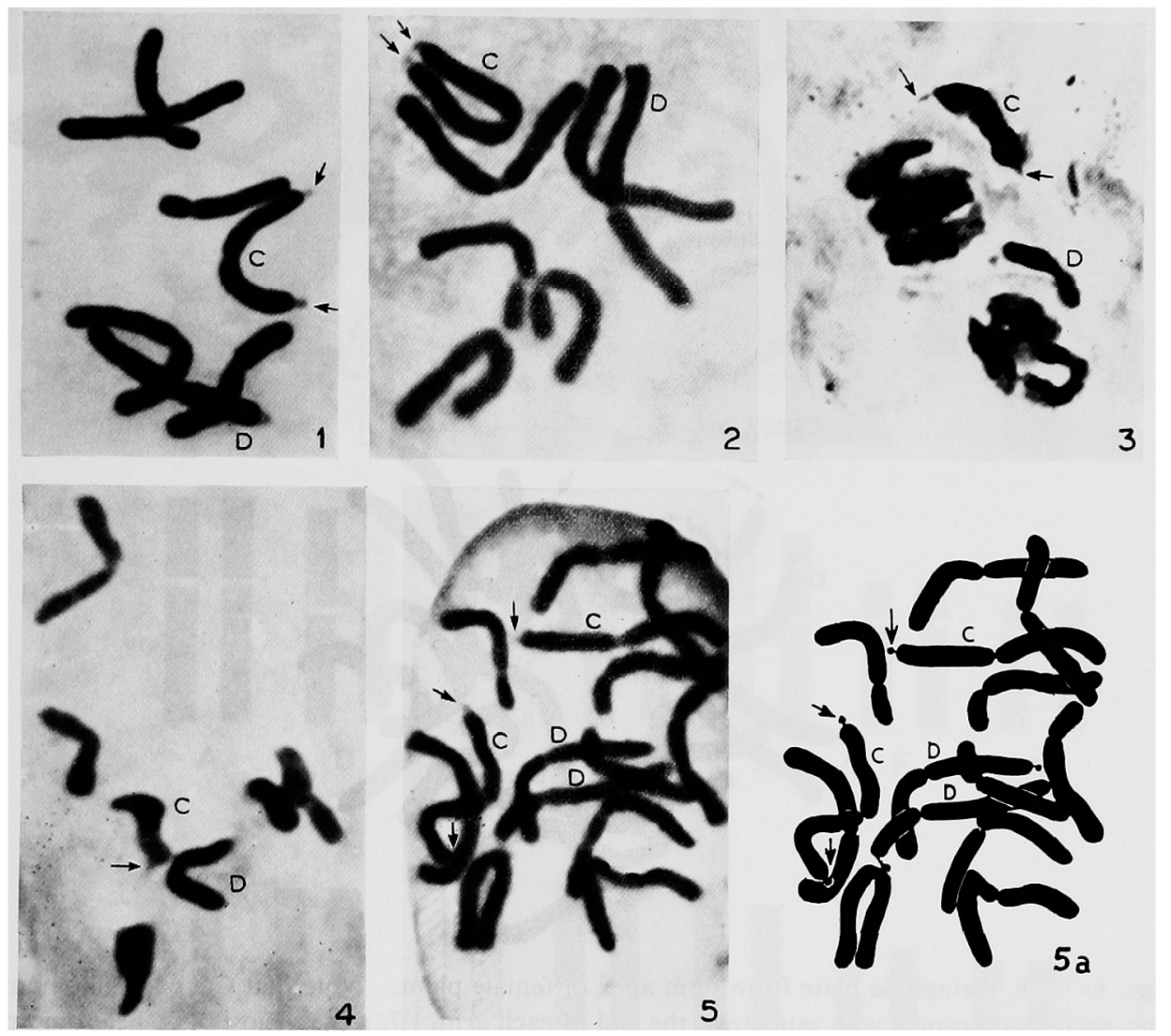

Figs. 1-5. 1 and 2, metaphase plates from two $q$ gametophytes. Note that the sex chromosome C has two satellites, one at the end of each arm, at arrows. 3, metaphase plate from female determining pollen. Note that the $\mathrm{C}$ chromosome has two satellites, one at the end of each arm. 4, metaphase plate from male determining pollen. Note that the $\mathrm{C}$ chromosome has only one satellite, at arrow. 5, metaphase plate from stem apex of male plant. Note that $\mathrm{CC}$ pair is heteromorphic, one homologue with two satellites and the other with one satellite, at arrows.

\section{Karyotype from stem-tip apices}

Stem-tip mitoses of the male and female plants have been studied to confirm the chromosomal differences in the two sexes.

The karyotypes from stem apices show the same basic karyotype of 5 pairs of long and 2 pairs of small chromosomes. The only point of difference is in respect of the presence of satellites on the Metacentric pair CC. 
The male plants have been observed to be heterozygous for satellites on the Metacentric pair. One of the homologues has a satellite on one arm only, while the other has two, one on each arm (Fig. 5).

The female plants on the other hand are homozygous i.e. the Metacentric pair CC has two satellites, one on each arm in both the homologues (Fig. 6).

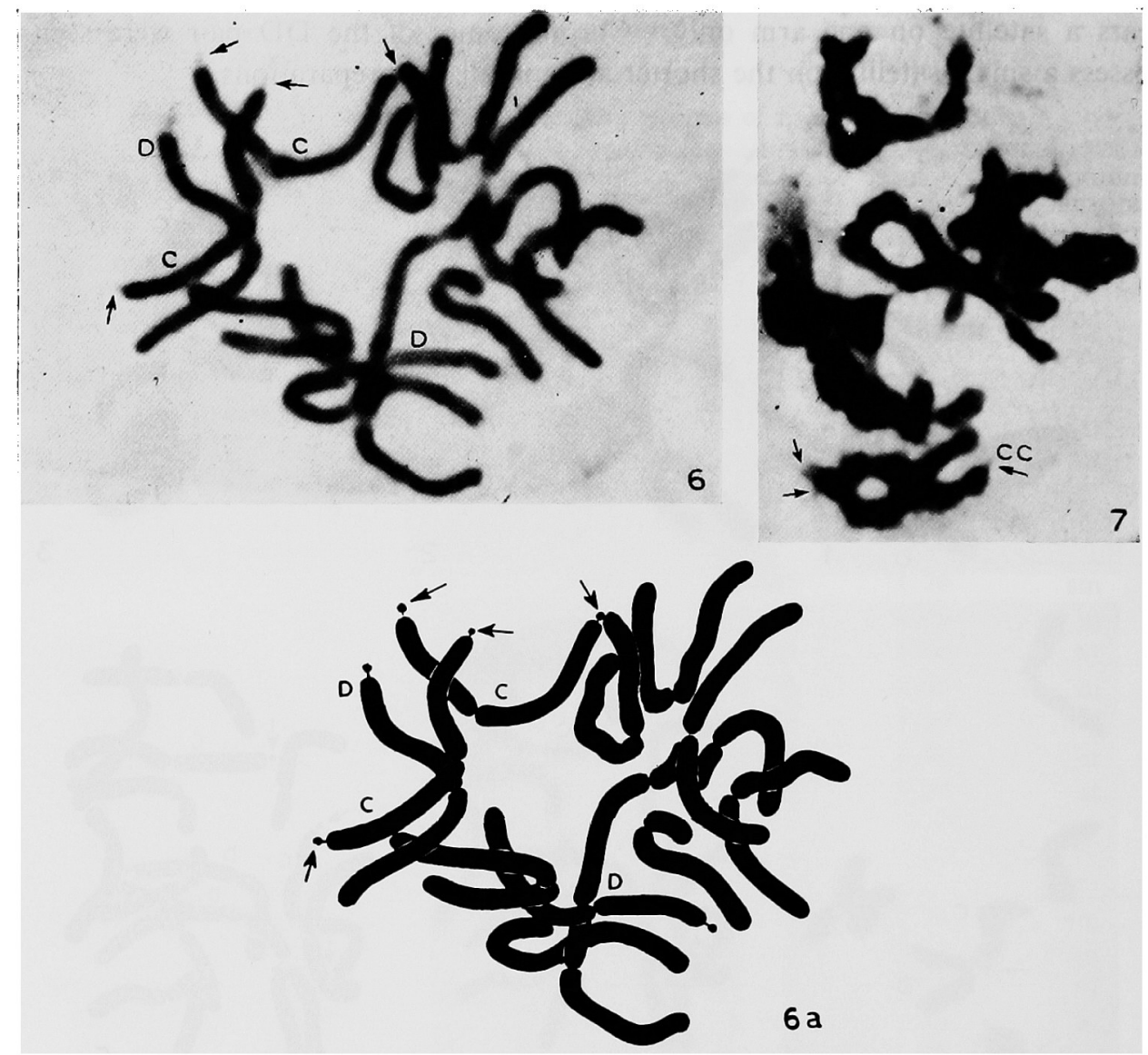

Figs. 6-7. 6, metaphase plate from stem apex of female plant. Note that CC pair is homomorphic, each chromosome with satellite at the end of each arm. 7, male meiosis. A heteromorphic bivalent $\mathrm{CC}$ is observed, one homologue with two satellites and the other with one satellite, at arrows. D chromosome in all cases is a sat-autosome.

\section{Male meiosis}

Further indications for the presence of the heteromorphic sex pair in the male $F$. foliata Boiss. are provided from a study of its meiosis in the pollen mother cells. The heteromorphic pair could be discerned at diplotene. In Fig. 7 the bivalent at 6 o'clock shows one homologue with a satellite on each arm, whereas the other shows it only on one arm.

\section{Discussion}

A karyotypic analysis from both gametophytic and sporophytic tissues of 
F. foliata Boiss. revealed that recognisable chromosomal differences exist between the two sexes (see Fig. 8). The karyogram of the female individuals shows that both members of the Metacentric pair CC are satellited on both arms, while that of the male individual shows that this same pair is heteromorphic, with only one of its members bearing satellite on each arm and the other with a satellite on one arm
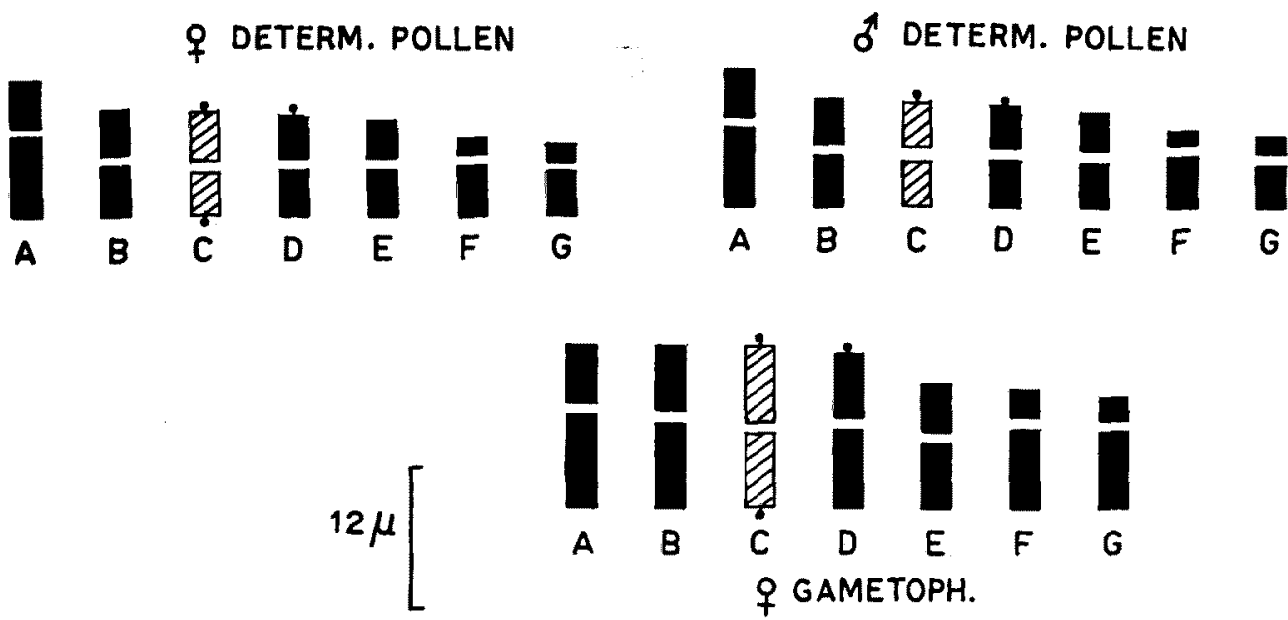

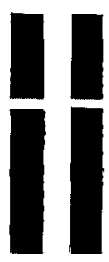

A

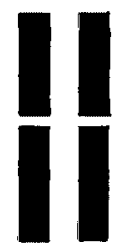

B

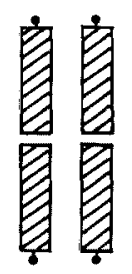

C

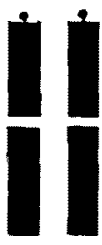

D

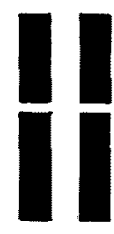

$E$

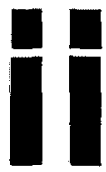

$\mathbf{F}$

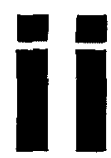

G \& STEM APEX

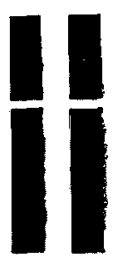

A

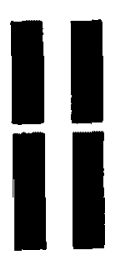

B

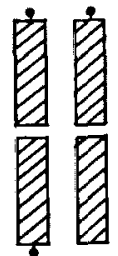

C

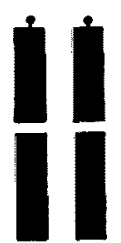

D

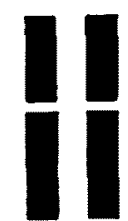

E

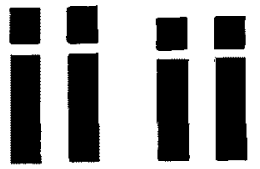

$\mathbf{F}$
G \& STEM APEX

8

Fig. 8. Idiograms from female determining pollen, male determining pollen, female gametophyte, and from stem apex of female and male plants respectively. Hatched chromosomes are sexchromosomes.

only. Further, the karyotype in the haploid set of the female gametophyte shows $\mathrm{C}$ chromosome always with two satellites, whereas the male gametophytes show a two fold picture of the $\mathrm{C}$ chromosomes. In some haploid complements, $\mathrm{C}$ chromosome possesses two satellites, while in others it bears only one satellite. 
It is interesting to recall here the work of Abraham and Mathew (1962) on the karyotype and sex chromosomes of Cycas pectinata and of Lee (1954) and Pollock (1957) on the karyotype and sex chromosome of Ginkgo biloba Linn. The situation in these is more or less similar to what has been found in $E$. foliata. The evidence is convincing that the sex mechanism in the present species also is related to the presence of satellites. In the female plants the two homologues of the Metacentric pair have two satellites one on each arm i.e. $\ddot{v} \ddot{v}$, whereas in the male plants one homologue has two satellites (one on each arm) while the other has it only on one arm i.e. $\ddot{V} \dot{V}$. The male sex is thus heterogametic with XY mechanism, while the female sex is homogametic with XX mechanism.

In the tetraploid species of the genus it often happens that three types of individuals are found, strictly $\delta$, strictly $ᄋ$, and those that are hermaphrodite bearing $\hat{\sigma}$ as well as $q$ cones. Such is the case for example in $E$. intermedia with $\mathrm{n}=14$ chromosomes. It is interesting to recapitulate what Mehra (1950) had written earlier in connection with the sexuality of individuals in this species. "The occurrence of sex chromosomes has not yet been established in any species of the genus Ephedra by cytological analysis, but the strict sexuality displayed by the male and female plants and the occurrence of hermaphrodite ones in individuals of the same species suggests that these individuals may be sex-controlled by the ratio of the X-chromosomes to autosomes in their nuclear constitution. The species may have come from immediate unisexual diploid ancestors in which sex was governed by sex chromosomes". In the present publication it has been brought to light that heteromorphic sex chromosome pair does exist in the diploid species (presently E. foliata) but the difference is minute and is based on the number of satellites on the CC chromosome pair.

It also seems remarkable that in all the three hitherto investigated species, Ginkgo biloba, Cycas pectinata and Ephedra foliata, belonging to diverse orders of the Gymnosperms, it is the satellites that determine the homologues for maleness or femaleness in the heteromorphic sex pair.

\section{Summary}

The karyotype of Ephedra foliata-a strictly dioecious species, has been studied from root tips, stem apices, female gametophyte and male gametophyte.

The haploid set consist of seven chromosomes in a $1 \mathrm{M}+3 \mathrm{~m}+1 \mathrm{sm}+2$ st arrangement i.e. $5 \mathrm{~V}^{\prime} \mathrm{s}+2 \mathrm{~J}$ 's. From among the $5 \mathrm{~V}$ 's two chromosomes one with Median point primary constriction (M) and one with a median region primary constriction (m) are satellited, the satellite in the latter case being on the short arm. Meiotic and karyotypic studies lend a strong evidence that the sex determining mechanism in this plant is related to satellites in the Metacentric pair CC. In female plants the two homologues of this Metacentric pair have two satellites one on each arm i.e. $\ddot{\mathrm{V}} \ddot{\mathrm{V}}$, whereas in the male plants one homologue has two satellites (one on each arm) while the other has it only on one arm i.e. $\ddot{V} \dot{V}$. 


\section{References}

Abraham, A. and Mathew, P. M. 1962. Cytological studies in Cycads: Sex chromosomes in Cycas. Ann. Bot. 26: 261-266.

Allen, C. E. 1917. A chromosome difference as correlated with sex differences in Sphaerocarpos. Science 46: 466-467.

Darlington, C. D. and LaCour, L. F. 1950. The Handling of Chromosomes. George Allen and Unwin Ltd. London.

Eichhorn, A. 1928. Sur la mitose somatique et le satellite du Ginkgo biloba. Compt. Rend. Soc. Biol. Paris 99: 1787-1788.

Lee, C. L. 1954. Sex chromosomes in Ginkgo biloba. Amer. J. Bot. 41 : 545-549.

Levan, A., Fredga, K. and Sandberg, Avery A. 1964. Nomenclature for centromeric position on chromosomes. Hereditas 52: 201-220.

Mehra, P. N. 1946. A study of the karyotypes and the occurrence of diploid male gametophyte in some species of the genus Ephedra. Proc. National Acad. Sci. India. Sec. B, 16: 259-286.

- 1950. Occurrence of hermaphrodite flowers and the development of female gametophyte in Ephedra intermedia Sprenk et Mey. Ann. Bot. NS 54: 165-180.

- 1971. Late male gametogenesis in the genus Ephedra. Annales Universite A.R.E.R.S. 9: 311-315.

Pollock, E. G. 1957. The sex chromosome of the maiden hair tree. J. Heredity 48: 290-293. 